\title{
An Epidermal Stimulation and Sensing Platform for Sensorimotor Prosthetic Control, Management of Lower Back Exertion, and Electrical Muscle Activation
}

\author{
Dr. Baoxing Xu, \\ Department of Mechanical and Aerospace Engineering, University of Virginia, Charlottesville, VA \\ 22904, USA; Department of Materials Science and Engineering Beckman Institute, and Frederick \\ Seitz Materials Research Laboratory, University of Illinois at Urbana-Champaign, Urbana, IL \\ 61801, USA
}

\section{Aadeel Akhtar,}

Neuroscience Program, Medical Scholars Program, Beckman Institute, and Coordinated Science Laboratory, University of Illinois at Urbana-Champaign, Urbana, IL 61801, USA

\section{Yuhao Liu,}

Department of Materials Science and Engineering Beckman Institute, and Frederick Seitz Materials Research Laboratory, University of Illinois at Urbana-Champaign, Urbana, IL 61801, USA

\section{Hang Chen, \\ Department of Engineering Mechanics and Center for Mechanics and Materials, Tsinghua University, Beijing 100084, China; Department of Mechanical Engineering and Department of Civil and Environmental Engineering, Center for Engineering and Health and Skin Disease Research Center, Northwestern University, Evanston, IL 60208, USA}

\section{Prof. Woon-Hong Yeo,}

Department of Materials Science and Engineering Beckman Institute, and Frederick Seitz Materials Research Laboratory, University of Illinois at Urbana-Champaign, Urbana, IL 61801, USA; Department of Mechanical and Nuclear Engineering, Center for Rehabilitation Science and Engineering, Virginia Commonwealth University, Richmond, VA 23284, USA

\section{Dr. Sung Park II,}

Department of Materials Science and Engineering Beckman Institute, and Frederick Seitz Materials Research Laboratory, University of Illinois at Urbana-Champaign, Urbana, IL 61801, USA

\section{Brandon Boyce,}

Department of Aerospace Engineering, Beckman Institute, and Coordinated Science Laboratory, University of Illinois at Urbana-Champaign, Urbana, IL 61801, USA

Correspondence to: John A. Rogers, jrogers@illinois.edu.

B.X., A.A., and Y.L. contributed equally to this work.

Supporting Information

Supporting Information is available from the Wiley Online Library or from the author. 


\section{Hyunjin Kim,}

Department of Materials Science and Engineering Beckman Institute, and Frederick Seitz Materials Research Laboratory, University of Illinois at Urbana-Champaign, Urbana, IL 61801, USA

Jiwoo Yu,

Department of Materials Science and Engineering Beckman Institute, and Frederick Seitz Materials Research Laboratory, University of Illinois at Urbana-Champaign, Urbana, IL 61801, USA

\section{Hsin-Yen Lai,}

Department of Materials Science and Engineering Beckman Institute, and Frederick Seitz Materials Research Laboratory, University of Illinois at Urbana-Champaign, Urbana, IL 61801, USA

\section{Sungyoung Jung,}

Department of Materials Science and Engineering Beckman Institute, and Frederick Seitz Materials Research Laboratory, University of Illinois at Urbana-Champaign, Urbana, IL 61801, USA

\section{Yuhao Zhou,}

Department of Materials Science and Engineering Beckman Institute, and Frederick Seitz Materials Research Laboratory, University of Illinois at Urbana-Champaign, Urbana, IL 61801, USA

\section{Dr. Jeonghyun Kim,}

Department of Materials Science and Engineering Beckman Institute, and Frederick Seitz Materials Research Laboratory, University of Illinois at Urbana-Champaign, Urbana, IL 61801, USA

\section{Seongkyu Cho,}

Department of Materials Science and Engineering Beckman Institute, and Frederick Seitz Materials Research Laboratory, University of Illinois at Urbana-Champaign, Urbana, IL 61801, USA

\section{Prof. Yonggang Huang,}

Department of Mechanical Engineering and Department of Civil and Environmental Engineering, Center for Engineering and Health and Skin Disease Research Center, Northwestern University, Evanston, IL 60208, USA

\section{Prof. Timothy Bretl, and}

Department of Aerospace Engineering, Beckman Institute, and Coordinated Science Laboratory, University of Illinois at Urbana-Champaign, Urbana, IL 61801, USA

\section{Prof. John A. Rogers}

Department of Materials Science and Engineering Beckman Institute, and Frederick Seitz Materials Research Laboratory, University of Illinois at Urbana-Champaign, Urbana, IL 61801, USA

John A. Rogers: jrogers@illinois.edu 
Skin-mounted sensors of physiological signals are useful in areas ranging from clinical diagnostics to human-machine interfaces. $\left.{ }^{\left[{ }^{1}-6\right.}\right]$ The recent development of concepts in "skinlike" semiconductor technologies, sometimes referred to as epidermal electronics, create important opportunities in long-term, noninvasive, conformal interfaces to the body. $\left.{ }^{7}{ }^{13}\right]$ These systems offer advantages in device mechanics and user mobility over traditional technologies for healthcare monitoring and disease diagnostics, with demonstrated capabilities in precision measurement of hydration, $\left[{ }^{[14}\right]$ strain,$\left.{ }^{[15}{ }^{17}\right]$ pressure $\left[{ }^{18}, 19\right]$ temperature, $\left.{ }^{[20}\right]$ and other parameters of interest. Additional recent work shows that similar platforms designed for the fingertips can offer advanced capabilities in electrotactile stimulation. ${ }^{\left[{ }^{2}\right]}$ This previous work focused, however, on materials and circuit design aspects without any demonstrated application. Combining these functions in a single, simple device platform designed for operation on the trunk or limbs of the body is attractive for neuromuscular electrical stimulation, $\left.{ }^{[22}\right]$ neuromodulation rehabilitation therapy, $\left.{ }^{23}\right]$ pain mitigation and prevention, $\left.{ }^{[24}\right]$ human-machine interfaces, ${ }^{[5]}$ and sensorimotor control in prosthetic and orthotic devices, $\left.{ }^{25}\right]$ where electromyography (EMG) and electrostimulation can serve as sensing and actuating platforms. Here, we present systems of this type, where multiple transcutaneous electrical stimulation electrodes cointegrate on a common substrate with sensors for electromyography, temperature, and mechanical strain. Abilities for simultaneous recording of physiological data and presentation of neural stimulatory inputs provide valuable functionality, as illustrated in examples of sensorimotor prosthetic control, management of lower back exertion, and electrical muscle activation.

Figure 1a provides an optical image of a multifunctional device constructed from patterned metal traces and polymer dielectric materials on a thin layer of silicone elastomer (thickness: $60 \mu \mathrm{m}$, Young's modulus: $\approx 60 \mathrm{kPa}$, Smooth-on, USA), supported by a temporary, watersoluble substrate of polyvinyl alcohol (PVA, Aicello, Japan). The layout includes electrodes for electrotactile stimulation and measurement of EMG signals, temperature, and strain, as in the schematic illustration of Figure 1b. The fabrication details appear in Note S1 (Supporting Information). The simplicity of the construction represents a key feature of the design. In particular, the active parts of the entire system involve only two layers of metal with patterned interlayer dielectrics; semiconductor materials are not required. The temperature sensor consists of a serpentine conductive trace $(\mathrm{Cr} / \mathrm{Au})$ with a width of $20 \mu \mathrm{m}$, thickness of $200 \mathrm{~nm}$, and total length of $26 \mathrm{~mm}\left(\approx 1 \mathrm{~mm}^{2}\right.$ total area), in which the temperature coefficient of resistance in the Au serpentine traces $\left(2.5 \times 10^{-3}{ }^{\circ} \mathrm{C}^{-1}\right)^{\left[{ }^{20}\right]}$ provides the basis for the measurement. The EMG sensor uses the same metal, patterned in a different geometry, for reference, measurement and ground electrodes that each consist of 20 $\mu \mathrm{m}$ wide circular concentric rings ( $2 \mathrm{~mm}$ width and $12 \mathrm{~mm}$ length; $24 \mathrm{~mm}^{2}$ sensor area) in an interwoven serpentine morphology to allow mechanical stretchability, and with a spacing of $\approx 10 \mathrm{~mm}$ to optimize signal quality. $\left.{ }^{26}\right]$ Each stimulation electrode is coaxial, consisting of an inner disk with a radius of $1.0 \mathrm{~mm}$, an outer ring with a radius of $2.0 \mathrm{~mm}$, and a 0.5 $\mathrm{mm}$ space in between. The strain sensor, also constructed in serpentine traces $(20 \mu \mathrm{m})$ with wide radii of curvature $(60 \mu \mathrm{m})$ in the same metal layer, offers uniaxial operation in a multigrid geometry configured in an orientation opposite to that of the temperature sensor, with a width of $20 \mu \mathrm{m}$, thickness of $200 \mathrm{~nm}$, and total length of $27 \mathrm{~mm}\left(\approx 1.4 \mathrm{~mm}^{2}\right.$ sensor area). This design minimizes the transverse sensitivity of the strain gauge and improves the 
accuracy of the measurement. A thin film of polyimide (PI, thickness: $1.2 \mu \mathrm{m}$; Young's modulus $\approx 2.5 \mathrm{GPa}$; Sigma-Aldrich) encapsulates the temperature and strain sensors. The width of the PI patterns is slightly larger, by $50 \mu \mathrm{m}$ on each side, than that of the electrodes, to relax tolerances on registration. This layout can accommodate any reduction in lateral dimensions associated with the reactive etching process. The PI also places the metal at the neutral mechanical plane, thereby minimizing the effects of bending strain on temperature. The EMG and stimulation electrodes remain exposed to the skin. All sensors connect to peripheral contact pads that allow interfaces to external power supplies and data acquisition hardware. Mounting on the skin involves washing away a water-soluble backing layer of PVA, using procedures described previously. ${ }^{[7]}$ Figure $1 \mathrm{c}$ illustrates the way in which the thin, soft construction of the device allows it to conform and adhere to the surface of the skin, based on van der Waals interactions alone. This low modulus mechanics also avoids any significant constraint on natural motions of the skin. The advantages of using this type of device compared to conventional gel-based electrodes for electrical stimulation or EMG measurement are summarized in Table $1 .{ }^{[27,28]}$ Stability of similar types of structures under cyclic strains is excellent. ${ }^{\left[{ }^{29}\right]}$ Long-term durability is confirmed by visual and functional evaluation over a two week period of continuous use. $\left.{ }^{[28}\right]$

The stimulation electrodes provide an important functional capability. Current injected into the skin through these electrodes stimulates nerve fibers to elicit sensations that resemble tingling, vibration, light touch, or pressure. $\left.{ }^{[30}\right]$ While the contact area of the stimulation electrodes in this epidermal device is much smaller than that of conventional electrodes, stimulation comfort can be maintained by selecting appropriate current amplitudes and pulse durations. A previous study using conventional hardware reported no discomfort from subjects who received $10 \mathrm{~h} \mathrm{~d}^{-1}$ electrotactile stimulation on the upper arm for two weeks. $\left.{ }^{[30}\right]$ A constant-current monophasic square pulse ( $3 \mathrm{~mA}$ amplitude, $0.2 \mathrm{~ms}$ pulse duration, $20 \mathrm{~Hz}$ frequency, as shown in Figure S1, Supporting Information) applied to the skin through electrode 2 using a computer-controlled linear isolated stimulator (STMISOLA, BIOPAC, Inc., CA) yields typical voltage responses, shown in Figure 2a, that are similar to those obtained with a commercial gel-based adhesive electrode (Neuroline 710, Ambu A/S, Denmark) (Figure S2, Supporting Information). The voltage response of a single pulse from the epidermal device is shown in Figure $2 \mathrm{~b}$. The differences in voltage waveforms between the conventional electrodes and our epidermal device are mainly due to their different area of contact with the skin. The voltage response across the anode and cathode of the stimulation electrodes (Figure S3, Supporting Information) that represents the electrode-skin interface and the underlying tissue can be captured. ${ }^{[30,31]} \mathrm{Here}, R_{\mathrm{es}}$ and $C_{\mathrm{es}}$ are the effective resistance and capacitance, respectively, of the electrode-skin interface. Procedures for computing these values appear in Note S2 (Supporting Information). $R_{\mathrm{es}}$ and $C_{\mathrm{es}}$ depend on the contact areas of the electrodes, their constituent materials, the thickness and electrical properties of the skin, and the electrical waveform. ${ }^{\left[{ }^{30}\right]}$ Experiments that involve stimulating the skin superficial to the left flexor carpi radialis and normalizing to the size of the electrode, yields values of $273.5 \mathrm{k} \Omega \mathrm{mm}^{2}$ and $75.13 \mathrm{nF} \mathrm{mm}{ }^{-2}$ for $R_{\mathrm{es}}$ and $C_{\mathrm{es}}$, respectively, for the epidermal device; and $30.79 \mathrm{M} \Omega \mathrm{mm}^{2}$ and $20.84 \mathrm{pF} \mathrm{mm}^{-2}$ for the conventional electrodes. The modest resistance associated with the epidermal device reduces 
the likelihood of local regions of high current density that can cause painful stimulation. $\left.{ }^{30}, 32\right]$

Due to the use of a common, compact device platform, the stimulation electrodes can cause artifacts in the EMG recordings, as shown in Figure 2c for the case of stimulation $(20 \mathrm{~Hz}$, $200 \mu$ s positive monophasic square pulse across stimulation channels 1 and 2 with an amplitude of $1.6 \mathrm{~mA}$ ) on the left flexor carpi radialis muscle of a 21 year old female subject during EMG recording for flexing of the wrist for $2 \mathrm{~s}$, resting for $3 \mathrm{~s}$, and then clenching of the hand for $2 \mathrm{~s}$, each to maximum voluntary contraction (MVC). Figure 2d shows the EMG signal collected without electrical stimulation. Such data are similar to those obtained using conventional sensors, as in Figure S4, Supporting Information. A digital comb filter can attenuate responses at frequencies at $20 \mathrm{~Hz}$ and its harmonics, ${ }^{[22]}$ to eliminate the stimulation artifact without significantly altering the EMG data, as illustrated in Figure 2c. Additional experiments show that the stimulation frequency, magnitude, number, and position of the electrodes do not affect the EMG measurements (Figure S4, Supporting Information).

Further study reveals the extent of coupling between the other active components of the device. Constant current stimulation, for example, can potentially lead to Joule heating in the skin, which affects the response of the temperature sensor. Infrared (IR) images (FLIR Systems, Inc., OR) in Figure 2e show temperature distributions before and after maximum stimulation (constant current, just below the pain threshold) for 2 min with a current of 1.54 $\mathrm{mA}$ through a single electrode. The changes in temperature are small and localized to the regions immediately adjacent to the simulation electrodes. Even when stimulating through two electrodes, the temperature change remains localized (Figure S5b, Supporting Information). Design choices also ensure independent operation of the strain and temperature sensors. For example, stretching the device along the $x$-axis by $\approx 21 \%$, leads to large and readily measurable changes in the response of the strain sensor, but only small changes in the temperature sensor, as shown in Figure 2f.

The mechanics of these structural designs can be captured by finite element analysis (FEA). As shown in Figure S5c (Supporting Information) (only half of the model is shown due to the symmetric structure), during electrotactile stimulation using two symmetrical electrodes, the temperature increases $\approx 0.002{ }^{\circ} \mathrm{C}$ and is localized to the region of the electrode. The temperature change in other regions of the device, for example, near the strain sensor, is less than $0.0001{ }^{\circ} \mathrm{C}$, which is consistent with the experimental measurements by an IR camera (Figure 2e and Figure S5, Supporting Information). The FEA results further confirm that only small strains appear in the temperature sensor (Note S3b, Supporting Information). As shown in Figure S6 (Supporting Information), with a 21\% stretching deformation, the maximum strain that occurs at the junction between the EMG electrodes and the interconnecting wires is less than $0.3 \%$, which is within the elastic regime for the gold, and far less than the $1 \%$ fracture strain for all other constituent materials in the device. In Figure S6b (Supporting Information), the FEA shows that strains along the $y$-axis of the temperature sensor are less than $0.04 \%$. The overall device design can accommodate even large deformations associated with bending deformation at the elbows and knees, in agreement with experimental measurements (Figure 2f). 
Advanced surgical techniques, such as targeted reinnervation, provide patients with upper limb amputations the ability to control a prosthetic limb using intuitive muscle commands that map to their missing limb, and to experience sensations perceived as originating from their missing limb. $\left.{ }^{33}, 34\right]$ Here, nerves that originally supplied the missing limb are rerouted to intact muscle and skin elsewhere in the body. Because the reinnervated muscle and skin sites often overlap with or are in close proximity to one another, existing, bulk electrode technologies cannot simultaneously record EMG and electrically stimulate the overlying skin for force and proprioceptive feedback, as a means to reduce the probability of prosthesis abandonment. $\left.{ }^{35}, 36\right]$ The devices introduced here, where sensors and actuators can be located closely adjacent to one another, with lithographic precision, create opportunities in this context. A simple demonstration involves devices applied to the right flexor carpi radialis and the extensor carpi radialis muscle groups of a 22 year old male subject. The root mean square (RMS) value of the EMG signal serves as proportional control on the grip aperture of a humanoid robot (Baxter, Rethink Robotics, MA). A sensor (25 lb Flexiforce, Tekscan, Inc., MA) mounted on the inside of gripper measures the force applied to a plastic bottle filled with water (Figure 3a and Figure S7, Supporting Information). In evaluations, the subject, blindfolded and acoustically shielded, attempts to grip the bottle, both with and without stimulation. With stimulation, the subject receives sensory input at a level proportional to the force measured by the sensor. The maximum stimulation, $2 \mathrm{~mA}$, in this case, corresponds to $27 \mathrm{~N}$, the maximum force available to the gripper. Figure $3 \mathrm{~b}$ shows that, without feedback, the subject cannot consistently close the gripper without causing the bottle to collapse (Movie S1, Supporting Information). With force feedback, the subject can successfully stop the grip at any desired level of gentle touch. Similar capabilities are possible with a wooden block used in place of the bottle (Figure S7, Supporting Information).

Stimulation can also provide proprioceptive feedback, as demonstrated in the control of both virtual arms presented on a computer screen and physical robotic arms. Here, two separate devices mount over the long head of the biceps brachii and the lateral head of the triceps brachii muscles (Figure 3c). In the virtual arm targeting task, the subject attempts to flex and extend the elbow of a single degree-of-freedom virtual arm to match the orientation of a target virtual arm presented onscreen. ${ }^{[37,38]}$ The virtual arm can move between $-60^{\circ}$ and $60^{\circ}$. During experiments, the subject grips a vertical handle and maintains his elbow in a fixed position at $90^{\circ}$ with respect to the humerus to remove any natural proprioceptive cues. Flexing or extending the elbow joint against the handle generates EMG signals (Figure 3d and Figure S8, Supporting Information). Linear discriminant analysis classifies these signals to virtual arm movements every $0.1 \mathrm{~s}$, according to previously reported procedures. $\left.{ }^{5}, 37\right]$ Evaluations involve four conditions: EMG control with conventional electrodes and no feedback; EMG control with conventional electrodes and stimulation feedback; EMG control with the epidermal device and no feedback; and EMG control with the epidermal device and electrotactile feedback. The feedback activates stimulation with electrodes 1 and 2 , that maps, using the tactile funneling illusion, $\left.{ }^{39}\right]$ the virtual arm angular range $\left(-60^{\circ}\right.$ to $60^{\circ}$ ) to different current levels (Figure 3e). In the tactile funneling illusion, when two stimulation electrodes are simultaneously active, a single sensation is perceived between the two electrodes. By modulating the stimulation current amplitudes, the location of the 
perceived sensation can be adjusted to any point between the two electrodes. As a result, any virtual arm angle can be mapped to a unique stimulation location perceived between the two electrodes. In turn, the subject interprets this stimulation location as the joint angle of the virtual arm. The experimental protocol appears in Figure S9 (Supporting Information). A training phase involves free control of the virtual arm for $60 \mathrm{~s}$, and then presentation with five target angles to match. After each attempt, the subject presses a button to reveal the actual angle of motion. Performance tests based on 25 random targets appear in Figure 3f, which shows a schematic illustration of the virtual arm, as well as the errors between the target angle and the subject's estimate for each of the four conditions. Feedback yields statistically significant improvements in performance (two-way analysis of variance (ANOVA), Tukey post hoc, $p<0.05$ ). In particular, the mean absolute errors for conditions without feedback are $37.2^{\circ}$ and $31.6^{\circ}$ with conventional and epidermal electrodes, respectively. With feedback, the errors decrease significantly to $17.8^{\circ}$ and $16.9^{\circ}$ with conventional electrodes and epidermal electrodes, respectively. These performance gains are similar to those found in other studies using sensory substitution (electrotactile, vibrotactile, or skin stretch) for proprioceptive feedback in modulating upper limb joint angles. $\left[{ }^{37}, 38,40\right]$ While the performance with the epidermal electronics offers lower error both with and without feedback compared to the conventional electrodes, the differences are statistically insignificant. Nevertheless, the ability to provide multiple points of stimulation while simultaneously recording EMG in a single device, as opposed to the multiple sets of conventional electrodes needed to enable sensorimotor control, represents a significant advantage.

Another mode of use that is relevant for prostheses exploits the temperature sensors to estimate muscle fatigue. ${ }^{\left[{ }^{41}\right]}$ Figure S10 (Supporting Information) shows EMG signal recorded from the flexor carpi radialis muscle as a subject holds a weight $(25 \mathrm{lb})$ for an extended period of time. The data show decreases in the amplitude of the EMG signal and increases in the temperature with time, both consistent with muscle fatigue.

These collective capabilities allow for efficient prosthetic interfaces, as demonstrated by control of the elbow joint of a robot, using EMG recorded from devices on the upper arm (Movie S2, Supporting Information). The control involves gripping a bottle, lifting it, and placing it back down on a table while blindfolded and acoustically shielded. Here, electrotactile stimulation provides touch and proprioceptive feedback (Movie S3, Supporting Information). Extensions of these designs to increased numbers and densities of electrotactile stimulation electrodes (Figure S11, Supporting Information) can improve the resolution and performance.

Another application opportunity is in the prevention of excessive muscle exertion due, for example, to improper lifting that can cause chronic lower back pain (Figure S12, Supporting Information). Here, a device mounted on the lower back can simultaneously monitor temperature, strain, and EMG as indicators of exertion, and provide stimulatory feedback to prevent overexertion/extension. As a demonstration of functionality relevant in this context, experiments indicate an ability to differentiate stoop motions from squat lifting. A device on the right lumbar paraspinal region (22 year old male, Figure S12, Supporting Information) records data as the subject performs ten repetitions of four different actions: (1) stooping and 
then standing without lifting a load; (2) stooping and then standing while lifting a $25 \mathrm{lb}$ load (Figure 4a,c); (3) squatting and then standing without lifting a load; and (4) squatting and then standing while lifting a $25 \mathrm{lb}$ load (Figure $4 \mathrm{~b}$,d). The mounting location and deformation in three different action states appear in Figure 4c-e. Figure 4f,g summarizes the EMG and strain measurements for actions (1)-(4). A notable increase in EMG activity for the loaded compared to the unloaded cases suggests an ability to detect loaded lifting. Stooping motions induce signature responses of the strain gauge, distinct from those associated with squatting. Placing thresholds on both the EMG and strain data affords an ability to detect stoop lifting and to trigger an electrotactile stimulation alert signal.

Muscle exertion can also be measured. Although both EMG and temperature can serve as indicators of muscle fatigue, literature suggests that EMG is a poor indicator of lower back muscle fatigue due to unstable motor unit recruitment in this region. $\left.{ }^{42}, 43\right]$ In this circumstance, the temperature sensor provides a reliable indicator of muscle exertion, associated with increases in metabolic reactions and blood flow that lead to corresponding increases in skin temperature. To test this capability, a subject lying prone on a table flexes his back until exhaustion while holding a $25 \mathrm{lb}$ weight at his chest. Figure $4 \mathrm{~h}$ shows that although the EMG signals from the lower back remain unchanged, the temperature clearly increases with time. This temperature response therefore provides additional functionality in the context of monitoring muscle fatigue. Together with the EMG sensor, the strain gauge, and electrotactile stimulators, this platform offers important functionality intervention for lower back exertion.

The stimulation electrodes can provide not only sensory input, but they can also, when operated at increased current levels, induce muscle contractions, of relevance to reanimating paralyzed limbs, evaluating neuromuscular function or training for strength in athletes. $\left.{ }^{44}\right]$ Figure 5a shows a modified device that incorporates larger muscle stimulation electrodes mounted over the long head of the subject's biceps brachii muscle. These electrodes adopt serpentine structures similar to those used for electrotactile stimulation, but they cover larger areas to allow stimulation of multiple motor units to elicit muscle contractions. As in the case of the electrotactile stimulation electrodes, these serpentine designs allow for largestrain deformation, which is critically important in avoiding constraints or interface stresses associated with motion. In this device, an EMG sensor lies between the stimulation electrodes for monitoring in experiments that involve bicep muscle contractions in a 22 year old male subject induced by applying a positive, monophasic, constant voltage square pulse through a computer-controlled linear isolated stimulator (STMISOLA, BIOPAC, Inc., CA). Incrementally increasing the amplitude of the voltage identifies the threshold for inducing muscle twitch (Movie S4, Supporting Information). Figure 5b shows the resulting neuromuscular voltage response, known as the M-wave, from $1 \mathrm{~Hz}$ stimulation at $50 \mathrm{~V}$ recorded from the EMG sensor. Figure 5c shows one period of a representative M-wave at 50, 30, and $10 \mathrm{~V}$. Reducing the voltage decreases the amplitude of the muscle contraction, leading to a reduction in the M-wave amplitude, and vice versa (Figure S13 and Movie S5, Supporting Information). The amplitude of the M-wave can be used to modulate the stimulation voltage to produce a desired contraction level. As a test of the ability to recover voluntary EMG during electrical muscle stimulation using the device, the subject attempts to contract his biceps during application of $1 \mathrm{~Hz}$ stimulation at $50 \mathrm{~V}$. Figure S14 (Supporting 
Information) shows the original EMG signal with stimulation artifacts and M-waves present, as well as the filtered EMG signal that recovers the voluntary neural response. Other studies achieve similar results, $\left.{ }^{22}\right]$ but only with large bulky electrodes for stimulation and recording, as opposed to the compact, thin, conformal profile of the devices introduced here. Additionally, the temperature of the skin can be measured using the same device to detect muscle fatigue induced by long-term electrical muscle stimulation. Figure $5 \mathrm{~d}$ shows that the temperature increases quickly with the onset of stimulation and then becomes constant due to the heat transfer with air as the muscle fatigues. The temperature provides an important index of stimulation-induced muscle fatigue, similar to fatigue detection on the forearm and the upper arm, and will be important in modulating input voltages during long-term usage of electrical muscle stimulation. Consequently, the device is able to use both M-wave and muscle fatigue information to enable fine control of muscle contractions in electrical muscle stimulation applications.

In conclusion, the conformal, multifunctional, epidermal devices reported here seamlessly integrate sensors for electromyography, temperature, and strain with electrical stimulation electrodes, in a simple architecture that incorporates only metal traces and dielectric layers. Demonstrated application possibilities include prosthetic control with sensory feedback, monitors, and stimulation signals related to lower back exertion, and electrical muscle stimulation with feedback control. Future work will investigate long-term application in clinical settings, with a focus on patients with upper limb amputations to simultaneously control and sense from their prosthetic devices. Other possibilities include accelerated rehabilitation for stroke patients through the use of electrical muscle stimulation to reanimate paralyzed limbs. In such cases, means for accommodating variations in stimulation parameters that occur with changes in the impedance of the electrode-skin interface over time will be important. $\left.{ }^{45}\right]$ All such applications can exploit emerging capabilities in wireless power transfer and radio control in skin-like formats. $\left.{ }^{2}, 7,46\right]$

\section{Experimental Section}

\section{Device Fabrication}

The fabrication (details in Note S1, Supporting Information) began with spin-coating a sacrificial layer of poly(methylmethacrylate) (PMMA, $100 \mathrm{~nm}$, MicroChem) onto a silicon wafer, followed by a layer of PI (1.2 $\mu \mathrm{m}$, Sigma-Aldrich). Sputter deposition then formed a bilayer of chromium/gold (Cr/Au, $5 \mathrm{~nm} / 200 \mathrm{~nm})$. Photolithography, wet-etching, and oxygen reactive ion etching steps defined patterns for the temperature, strain, and EMG as well as the electrical muscle and electrotactile stimulation anodes. A second PI film was then coated and patterned as an insulating layer for additional bilayer of metal $(\mathrm{Cr} / \mathrm{Au}, 7$ $\mathrm{nm} / 300 \mathrm{~nm}$ ). Similar photolithography, wet-etching, and oxygen reactive ion-etching steps defined the stimulation cathodes. A third patterned layer of PI served to encapsulate the interconnects, the temperature and strain sensors, and the contacts. Dissolution of the PMMA allowed the entire device to be retrieved onto a water-soluble tape (3M). Electron beam evaporation of a bilayer of chromium/silica $\left(\mathrm{Cr} / \mathrm{SiO}_{2}, 5 \mathrm{~nm} / 60 \mathrm{~nm}\right)$ formed an exposed oxide for bonding to the surface of a thin film $(\approx 0.5 \mathrm{~mm})$ of silicone (Ecoflex, Smooth-on, Inc.) pretreated by exposure to ozone induced by ultraviolet light and coated on a substrate 
of PVA (Aicello, Toyohashi). Anisotropic conductive films (Elform) served as flexible electrical cable connections to contact pads on the device, for data acquisition and power supply.

\section{EMG Signals Measurement}

The EMG signals were recorded using a 16-channel EMG acquisition system (DelSys, Inc., MA) and amplified and band-pass filtered through an SA Instrumentation Isolated Bioamplifier (James Long, NY) along with a National Instruments DAQ. When measuring EMG, the middle EMG port on the epidermal device served as a ground electrode for obtaining a high quality signal. The other two ports provided interfaces for the differential recording electrode pair. The gain of the amplifier was set to 1000 . Specially designed MATLAB codes allowed removal of stimulation artifacts from the EMG signal.

\section{Strain Measurement}

A four-channel National Instruments Digital Multimeter was used to record the electrical resistance. The initial resistance, $R_{0}$, of the strain sensor was measured on the unstrained device mounted on the skin. Upon application of strain, the resistance, $R$, was recorded as a function of time. From the resistance, the strain could be computed, according to $\varepsilon=R-$ $R_{0} / R_{0} / G$, where $G$ is the gauge factor ( $\approx 2.6$ for the cases examined here). The effects of changes in temperature on the relative resistance during deformation were negligible, as shown in Figure 2.

\section{Temperature Measurement}

The same system for measuring strain was used to measure temperature. The change in temperature was extracted from the difference between the measured resistance, $R$, and the initial resistance, $R_{0}$, at the initial temperature $T_{0}$, according to $\Delta T=T-T_{0}=a\left|R-R_{0}\right|$, where $a$ is the thermal coefficient $\left(0.25 \mathrm{~K} \Omega^{-1}\right.$ for the cases examined here). The temperature data acquisition involved a National Instrument PXI-6289 board with custom software interface. The 16 bit A/D converter provided a resolution of $0.02{ }^{\circ} \mathrm{C}$ at a sampling frequency of $66.67 \mathrm{~Hz}$. The standard deviation of the temperature measurement over a period of $150 \mathrm{~s}$ was $0.021{ }^{\circ} \mathrm{C}$ for the sensor on skin. $\left.{ }^{[20}\right]$

\section{Current and Voltage Stimulation}

Electrical signals for stimulation were provided by a computer-controlled National Instruments digital to analog converter (NI-myDAQ) interfaced to an isolated linear stimulator (BIOPAC System, Inc., CA). The current or voltage stimulation waveforms were adjustable using specially designed software in MATLAB. Forearm, biceps, triceps, and lower back muscles were all successfully stimulated using this system.

\section{Experiments on Human Subjects}

All experiments on humans were conducted under approval from Institutional Review Board at the University of Illinois at Urbana-Champaign (protocol number: 13920). There are 3 subjects (age: 20-22, 1 female, 2 male). Research was carried out with informed signed consent from the subjects. 


\section{Supplementary Material}

Refer to Web version on PubMed Central for supplementary material.

\section{Acknowledgments}

This work was supported in part by Systems on Nanoscale Information fabriCs (SONIC), one of the six SRC STARnet Centers, sponsored by MARCO and DARPA. Baoxing Xu was supported by Beckman Institute Postdoctoral Fellowship at UIUC and start-up funds at the University of Virginia. Aadeel Akhtar and Timothy Bretl were supported by NSF 0955088 and NIH 1F30HD084201.

\section{References}

1. Ramanathan C, Ghanem RN, Jia P, Ryu K, Rudy Y. Nat Med. 2004; 10:422. [PubMed: 15034569]

2. Xu S, Zhang Y, Jia L, Mathewson KE, Jang KI, Kim J, Fu H, Huang X, Chava P, Wang R, Bhole S, Wang L, Na YJ, Guan Y, Flavin M, Han Z, Huang Y, Rogers JA. Science. 2014; 344:70. [PubMed: 24700852]

3. Budinger TF. Annu Rev Biomed Eng. 2003; 5:383. [PubMed: 14527317]

4. Tee BCK, Wang C, Allen R, Bao Z. Nat Nanotechnol. 2012; 7:825. [PubMed: 23142944]

5. Jeong JW, Yeo WH, Akhtar A, Norton JJS, Kwack YJ, Li S, Jung SY, Su Y, Lee W, Xia J, Cheng H, Huang Y, Choi WS, Bretl T, Rogers JA. Adv Mater. 2013; 25:6839. [PubMed: 24327417]

6. Oskoei MA, Hu H. Biomed Sign Process Control. 2007; 2:275.

7. Kim DH, Lu N, Ma R, Kim YS, Kim RH, Wang S, Wu J, Won S, Tao H, Islam A, Yu KJ, Kim T, Chowdhury R, Ying M, Xu L, Li M, Chung HJ, Keum H, McCormick M, Liu P, Zhang YW, Omenetto FG, Huang Y, Coleman T, Rogers JA. Science. 2011; 333:838. [PubMed: 21836009]

8. Kim DH, Lu N, Ghaffari R, Kim YS, Lee SP, Xu L, Wu J, Kim RH, Song J, Liu Z, Viventi J, de Graff B, Elolampi B, Mansour M, Slepian MJ, Hwang S, Moss JD, Won SM, Huang Y, Litt B, Rogers JA. Nat Mater. 2011; 10:316. [PubMed: 21378969]

9. Kim DH, Ghaffari R, Lu N, Wang S, Lee SP, Keum H, D’Angelo R, Klinker L, Su Y, Lu C, Kim YS, Ameen A, Li Y, Zhang Y, de Graff B, Hsu YY, Liu Z, Ruskin J, Xu L, Lu C, Omenetto FG, Huang Y, Mansour M, Slepian MJ, Rogers JA. Proc Natl Acad Sci USA. 2012; 109:19910. [PubMed: 23150574]

10. Ramuz M, Tee BCK, Tok JBH, Bao Z. Adv Mater. 2012; 24:3223. [PubMed: 22641411]

11. Son D, Lee J, Qiao S, Ghaffari R, Kim J, Lee JE, Song C, Kim SJ, Lee DJ, Jun SW, Yang S, Park M, Shin J, Do K, Lee M, Kang K, Hwang CS, Lu N, Hyeon T, Kim DH. Nat Nanotechnol. 2014; 9:397. [PubMed: 24681776]

12. Jeong GS, Baek DH, Jung HC, Song JH, Moon JH, Hong SW, Kim IY, Lee SH. Nat Commun. 2012; 3:977. [PubMed: 22864570]

13. Kaltenbrunner M, Sekitani T, Reeder J, Yokota T, Kuribara K, Tokuhara T, Drack M, Schwodiauer R, Graz I, Bauer-Gogonea S, Bauer S, Someya T. Nature. 2013; 499:458. [PubMed: 23887430]

14. Huang X, Liu Y, Cheng H, Shin WJ, Fan JA, Liu Z, Lu CJ, Kong GW, Chen K, Patnaik D, Lee SH, Hage-Ali S, Huang Y, Rogers JA. Adv Funct Mater. 2014; 24:3846.

15. Pang C, Lee GY, Kim TI, Kim SM, Kim HN, Ahn SH, Suh KY. Nat Mater. 2012; 11:795. [PubMed: 22842511]

16. Yamada T, Hayamizu Y, Yamamoto Y, Yomogida Y, Izadi-Najafabadi A, Futaba DN, Hata K. Nat Nanotechnol. 2011; 6:296. [PubMed: 21441912]

17. Lu N, Lu C, Yang S, Rogers J. Adv Funct Mater. 2012; 22:4044.

18. Wang C, Hwang D, Yu Z, Takei K, Park J, Chen T, Ma B, Javey A. Nat Mater. 2013; 12:899. [PubMed: 23872732]

19. Lipomi DJ, Vosgueritchian M, Tee BCK, Hellstrom SL, Lee JA, Fox CH, Bao Z. Nat Nanotechnol. 2011; 6:788. [PubMed: 22020121] 
20. Webb RC, Bonifas AP, Behnaz A, Zhang Y, Yu KJ, Cheng H, Shi M, Bian Z, Liu Z, Kim YS, Yeo WH, Park JS, Song J, Li Y, Huang Y, Gorbach AM, Rogers JA. Nat Mater. 2013; 12:938. [PubMed: 24037122]

21. Ying M, Bonifas AP, Lu N, Su Y, Li R, Cheng H, Ameen A, Huang Y, Rogers JA. Nanotechnology. 2012; 23:344004. [PubMed: 22885907]

22. Frigo C, Ferrarin M, Frasson W, Pavan E, Thorsen R. J Electromyogr Kinesiol. 2000; 10:351. [PubMed: 11018444]

23. Gerasimenko Y, Gorodnichev R, Moshonkina T, Sayenko D, Gad P, Edgerton VR. Ann Phys Rehabil Med. 1989; 52:53.

24. Loeser JD, Black RG, Christman A. J Neurosurg. 1975; 42:308. [PubMed: 1117329]

25. Antfolk C, D’Alonzo M, Rosén B, Lundborg G, Sebelius F, Cipriani C. Expert Rev Med Devices. 2013; 10:45. [PubMed: 23278223]

26. Young AJ, Hargrove LJ, Kuiken TA. IEEE Trans Biomed Eng. 2011; 58:2537. [PubMed: 21659017]

27. Green RA, Baek S, Poole-Warren LA, Martens PJ. Sci Technol Adv Mater. 2010; 11:014107.

28. Yeo WH, Kim YS, Lee J, Ameen A, Shi L, Li M, Wang S, Ma R, Jin SH, Kang Z, Huang Y, Rogers JA. Adv Mater. 2013; 25:2773. [PubMed: 23440975]

29. Dagdeviren C, Yang BD, Su Y, Tran PL, Joe P, Anderson E, Xia J, Doraiswamy V, Dehdashti B, Feng X, Lu B, Poston R, Khalpey Z, Ghaffari R, Huang Y, Slepian MJ, Rogers JA. Proc Natl Acad Sci USA. 2014; 111:1927. [PubMed: 24449853]

30. Kaczmarek KA, Webster JG, Bach-y-Rita P, Tompkins WJ. IEEE Trans Biomed Eng. 1991; 38:1. [PubMed: 2026426]

31. Long-Fei W, Jing-Quan L, Bin Y, Chun-Sheng Y. IEEE Sens J. 2012; 12:2898.

32. Keller T, Kuhn A. J Autom Control. 2008; 18:35.

33. Kuiken TA, Li G, Lock BA, Lipschutz RD, Miller LA, Stubblefield KA, Englehart K. J Am Med Assoc. 2009; 301:619.

34. Kuiken TA, Marasco PD, Lock BA, Harden RN, Dewald JPA. Proc Natl Acad Sci USA. 2007; 104:20061. [PubMed: 18048339]

35. Atkins DJ, Heard DCY, Donovan WH. J Prosth Orth. 1996; 8:2.

36. Peerdeman B, Boere D, Witteveen H, Veld RHit, Hermens H, Stramigioli S, Rietman H, Veltink P, Misra S. J Rehabil Res Dev. 2011; 48:719. [PubMed: 21938658]

37. Akhtar, A.; Nguyen, M.; Wan, L.; Boyce, B.; Slade, P.; Bretl, T. Haptics: Neuroscience, Devices, Modeling, and Applications. Auvray, M.; Duriez, C., editors. Vol. 8619. Springer; Berlin: 2014. p. 120

38. Wheeler J, Bark K, Savall J, Cutkosky M. IEEE Trans Neural Syst Rehabil Eng. 2010; 18:58. [PubMed: 20071271]

39. Nohama P, Lopes AV, Cliquet A. Artif Organs. 1995; 19:225. [PubMed: 7779010]

40. Witteveen HJB, Droog EA, Rietman JS, Veltink PH. IEEE Trans Biomed Eng. 2012; 59:2219. [PubMed: 22645262]

41. Tkach D, Huang H, Kuiken T. J NeuroEng Rehabil. 2010; 7:1. [PubMed: 20064261]

42. Farina D, Gazzoni M, Merletti R. J Electromyogr Kinesiol. 2003; 13:319. [PubMed: 12832163]

43. Yoshitake Y, Ue H, Miyazaki M, Moritani T. Eur J Appl Physiol. 2001; 84:174. [PubMed: 11320632]

44. Maffiuletti N, Minetto M, Farina D, Bottinelli R. Eur J Appl Physiol. 2011; 111:2391. [PubMed: 21866361]

45. Akhtar, A.; Boyce, B.; Bretl, T. Haptics Symp (HAPTICS). IEEE; Piscataway, NJ, USA: 2014. p. 69

46. Huang X, Liu Y, Chen K, Shin WJ, Lu CJ, Kong GW, Patnaik D, Lee SH, Cortes JF, Rogers JA. Small. 2014; 10:3083. [PubMed: 24706477]

Adv Mater. Author manuscript; available in PMC 2016 June 08. 
a
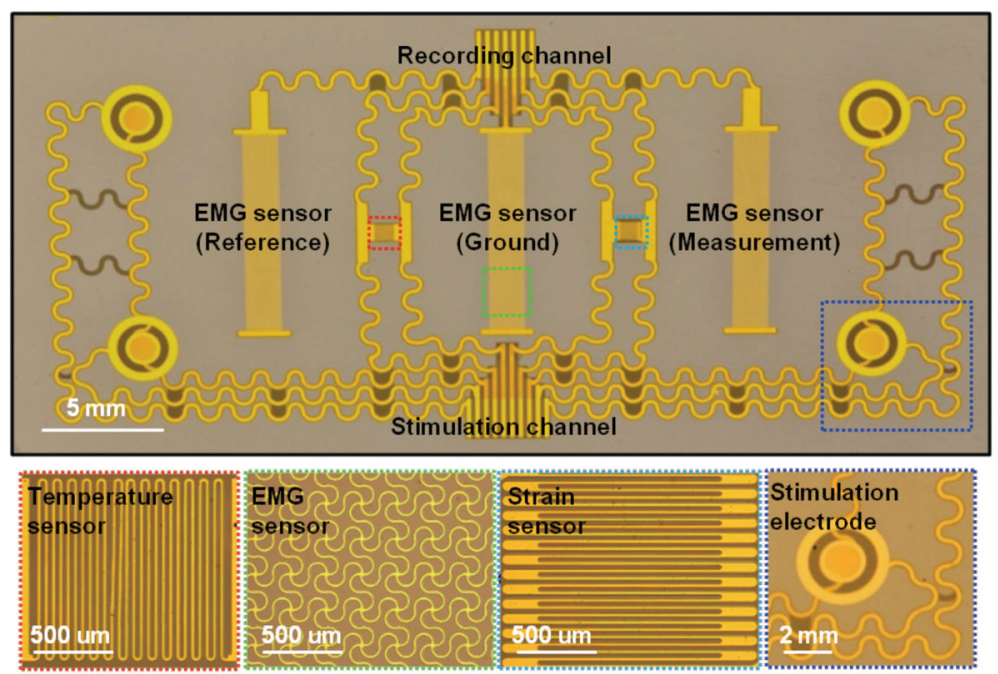

b

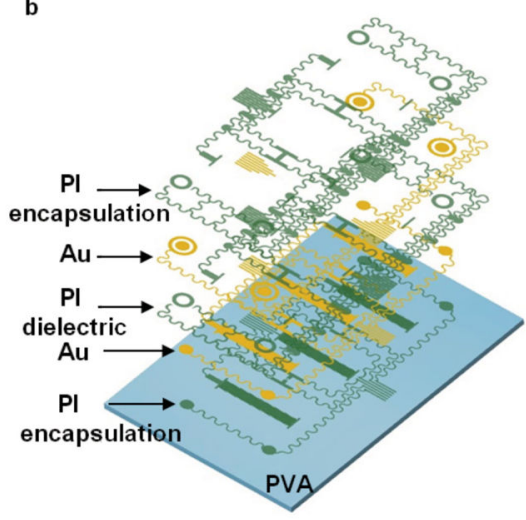

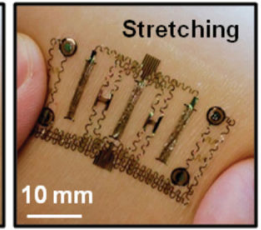

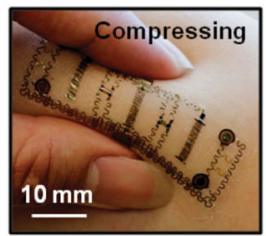

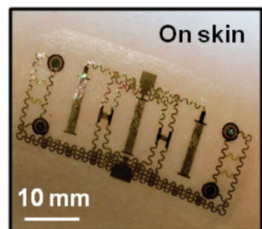

Figure 1.

Images and design features of a simple, multifunctional device with skin-like physical characteristics and capabilities in both sensing and stimulation. a) Planar view optical image of a representative device. The insets highlight various active regions. b) Exploded-view schematic illustration of the multilayer construction, composed only of patterns of metals and dielectrics. c) Images of a device mounted on the forearm, with examples under stretching, compressing, and peeling-off. 
a

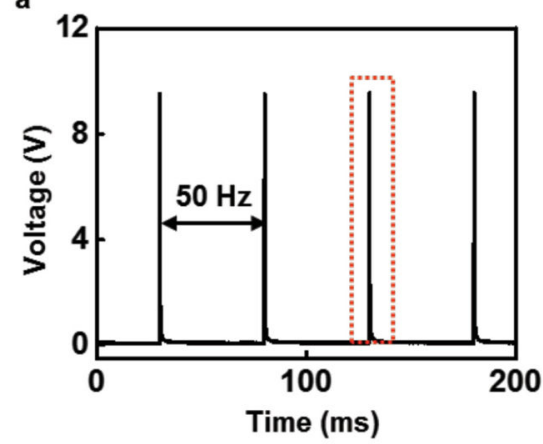

c

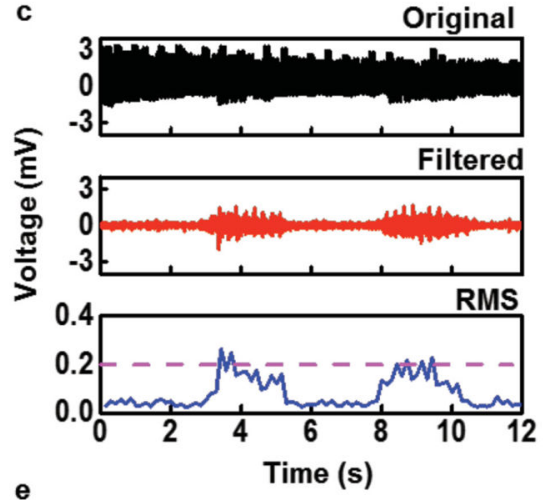

b

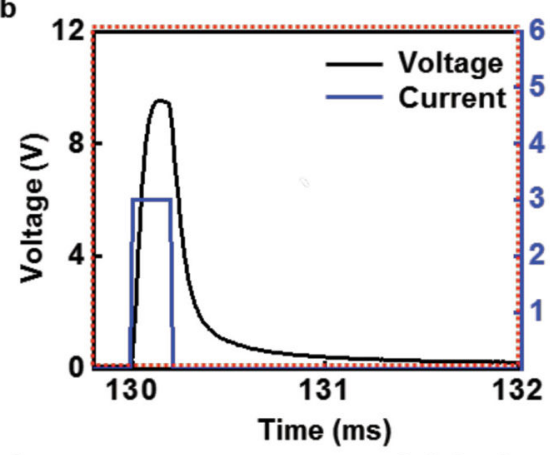

d

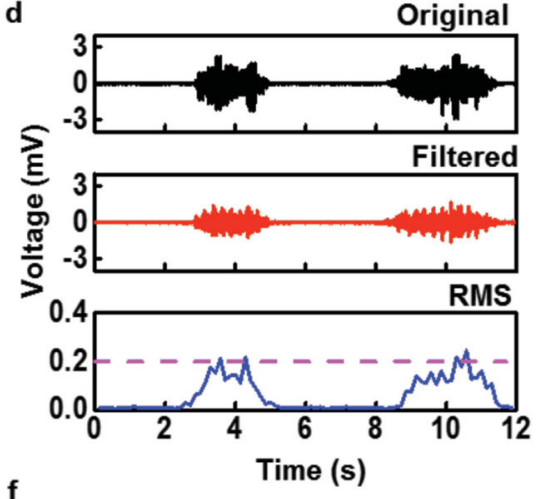

Temperature $\left({ }^{\circ} \mathrm{C}\right)$

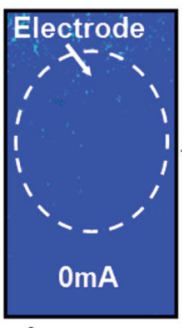

$\underline{1 \mathrm{~mm}}$
30.5

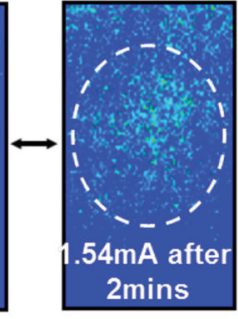

30.0

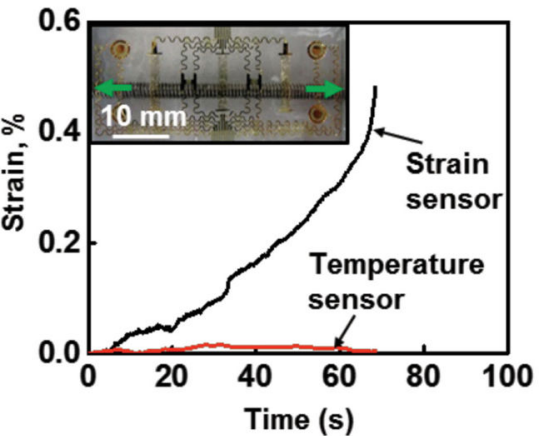

Figure 2.

Summary of functional attributes in sensing and stimulation. a) Voltage recorded between the two coaxial electrodes associated with an electrotactile stimulator during $20 \mathrm{~Hz}$ operation at $3 \mathrm{~mA}$. b) Magnified view of the recorded voltage over one period of stimulation (black), with applied stimulation current signal (blue). c) EMG signals collected from the forearm (original, filtered, and root mean square (RMS)) during simultaneous stimulation through top two electrodes (Figure 1a). The raw recorded data include signals that arise from the stimulation, which mask the EMG response. d) Optimized digital filters can remove the effects of stimulation, to yield EMG data that correspond well to recordings performed without stimulation. e) Infrared images of a stimulation electrode on the skin before (left) and after (right) 2 min of $20 \mathrm{~Hz}$ operation at $1.54 \mathrm{~mA}$. f) Mechanical analysis of the effect of stretch along the $x$-direction, as measured by the epidermal strain gauge, on temperature measurements performed simultaneously. The inset shows the epidermal device being stretched by a mechanical stage. 
a
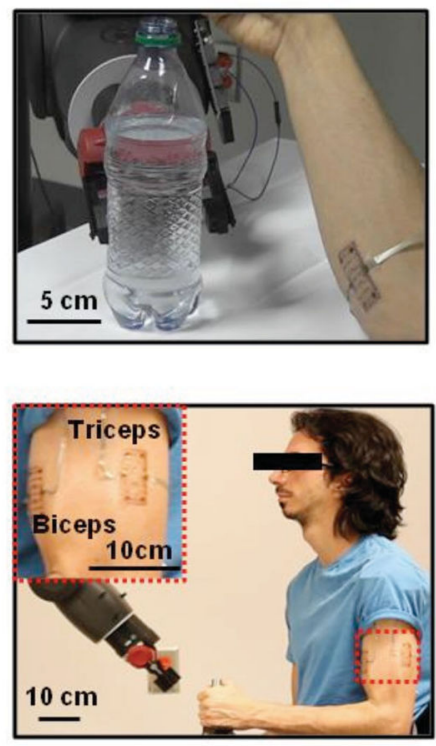

e

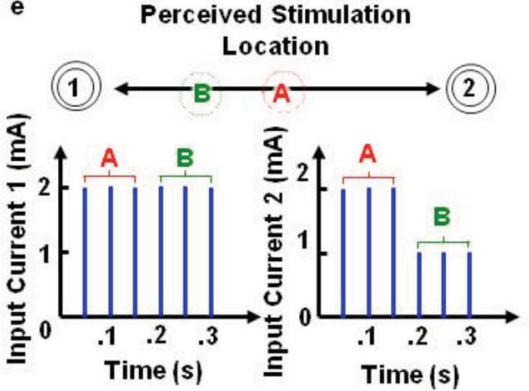

b
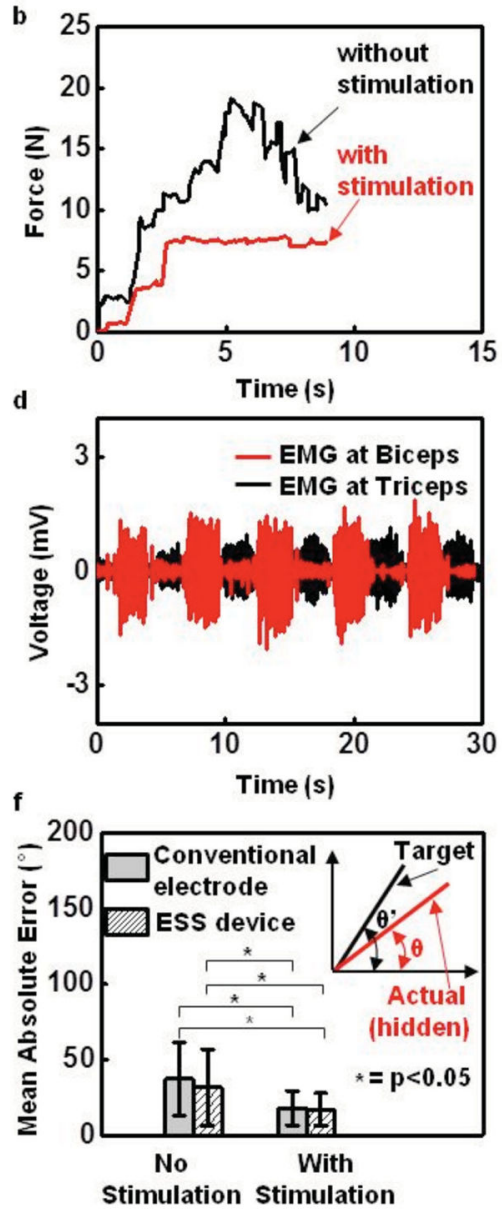

Figure 3.

Sensorimotor control of a robot arm. a) Image of a device on the forearm while controlling a robot arm to grip a bottle filled with water. b) Gripping force with and without stimulation feedback. When feedback is present, the subject can grip the bottle in a controlled manner, to prevent collapse. c) Image of devices on the bicep and tricep (inset) during control of the angle of the elbow of a robot arm. d) EMG signals from two devices when alternating between flexion and extension of the robot arm's elbow angle. e) Stimulation waveforms used to produce the tactile funneling illusion. When two stimulation electrodes are simultaneously active, sensation is perceived between the two electrodes. By modulating the stimulation current amplitudes, the location of the perceived sensation can be adjusted to any point between the two electrodes, as shown for current amplitudes A and B in the second electrode. The perceived sensation will be felt closer to the electrode with the higher current amplitude. f) Accuracy associated with the virtual arm targeting task with and without stimulation using both conventional electrodes and the epidermal device $(p<0.05$, one-way ANOVA, Tukey post hoc). The hidden virtual arm (inset, red line) was controlled via EMG to match a static visible target joint angle (inset, black line). When stimulation was present, the joint angle of the hidden virtual arm was mapped linearly to a location between two stimulation electrodes via the tactile funneling location. 
a

Figure 4.

Assessment of exertion and posture of the lower back during lifting. Cartoon illustration of motions associated with a) stoop lifting and b) squat lifting. Image of a device mounted on the lower back muscle groups and the deformation of device under c) stoop position with 25 lb weight, d) squat position with $25 \mathrm{lb}$ weight, and e) stand position with $25 \mathrm{lb}$ weight. f) Comparison of EMG and strain during stooping (red) and standing (black) with and without a $25 \mathrm{lb}$ load (top and bottom, respectively). g) Comparison of EMG and strain during squatting (red) and standing (black) with and without a $25 \mathrm{lb}$ load (top and bottom, respectively). h) Simultaneously recorded lower back EMG-RMS and temperature signals during muscle fatigue.

Adv Mater. Author manuscript; available in PMC 2016 June 08. 

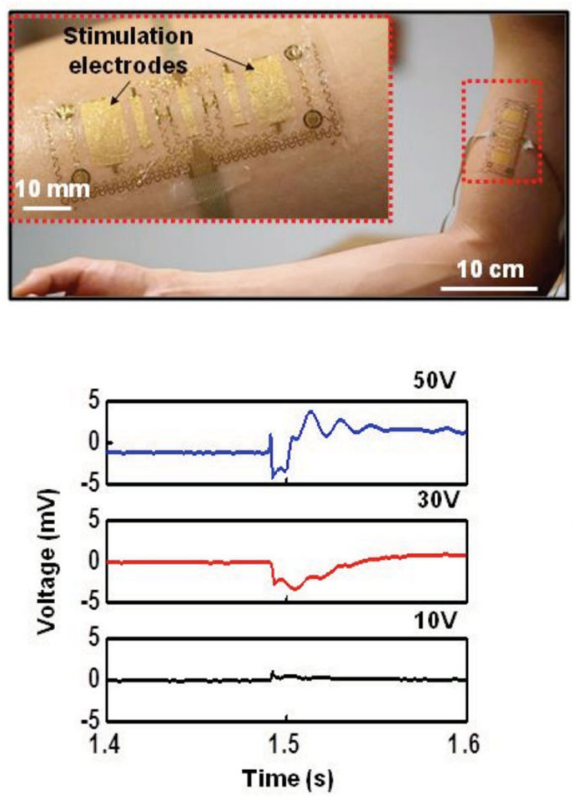

b

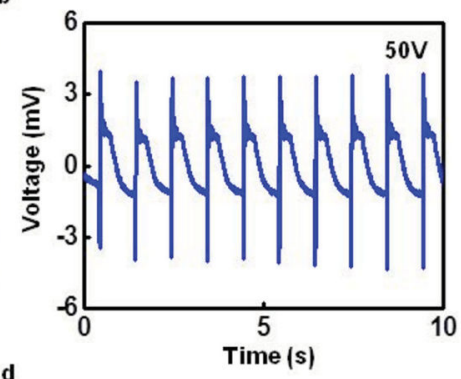

d

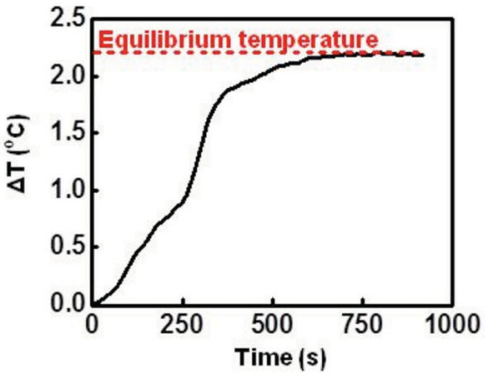

Figure 5.

Device with large-area electrodes for electrical muscle stimulation. a) Image of a device mounted on the biceps (magnified view, inset). b) Example of evoked M-waves from induced contraction of the biceps during $1 \mathrm{~Hz}$ stimulation at a voltage of $50 \mathrm{~V}$. c) Magnified view of a resulting $\mathrm{M}$-wave at 50, 30, and $10 \mathrm{~V}$ stimulations. d) Measured temperature from epidermal device during prolonged electrical muscle stimulation for $15 \mathrm{~min}$. 
Table 1

Comparison of conventional gel-based single electrode and EES multifunctional device.

\begin{tabular}{lcc}
\hline & Gel-based electrode $\left.{ }^{a}\right)$ & Multifunctional EES device \\
\hline Functionalities & EMG, stimulation & EMG, stimulation, temperature, strain \\
Modulus & $\left.\approx 200-300 \mathrm{kPa}^{27}\right]$ & $\approx 69 \mathrm{kPa}^{\left.2{ }^{28}\right]}$ \\
Thickness & $\approx 1 \mathrm{~mm}$ & $\approx 20 \mu \mathrm{m}$ \\
Scalability & Separate electrode pairs per EMG/stimulation channel & Integrated electrodes and sensors on a single substrate \\
Materials & $\mathrm{Ag} / \mathrm{AgCl}$ hydrogel & Silicone elastomer \\
\hline
\end{tabular}

${ }^{\text {a) }}$ Characteristics of gel-based electrode refer to Ambu Neuroline 700. 